\title{
Sensitivity of routine coagulation assays to direct oral anticoagulants: patient samples versus commercial drug- specific calibrators
}

\author{
Ming Sheng Lim ${ }^{1}$, Kent Chapman ${ }^{2}$, Priscilla Swanepoel ${ }^{2}$ and \\ AnOop K. EnJETI ${ }^{1,2,3,4,5}$ \\ ${ }^{1}$ Haematology Department, Calvary Mater Hospital, Waratah, ${ }^{2}$ Pathology North Hunter, \\ NSW Pathology, ${ }^{3}$ University of Newcastle, ${ }^{4}$ Hunter Cancer Research Alliance, and ${ }^{5}$ Hunter \\ Medical Research Institute, Newcastle, NSW, Australia
}

\begin{abstract}
Summary
Most studies on the sensitivities of coagulation assays to direct oral anticoagulants (DOACs) are based on norma plasma spiked with anticoagulant in the laboratory. Recent studies have shown that reagent sensitivity varies significantly depending on whether spiked or patient samples are used. The aim of this study was to compare the sensitivities of routine coagulation assays in patient samples and commercial drug specific calibrators using commonly used activated partial thromboplastin time (APTT) and prothrombin time (PT) reagents (i.e., Actin FS and Neoplastine $\mathrm{Cl}$ Plus for APTT and PT, respectively) in Australian laboratories. Samples collected at Pathology North Hunter $(\mathrm{PN}-\mathrm{H})$ for dabigatran $(n=39)$, rivaroxaban, $(n=56)$ or apixaban levels $(n=22)$ between February 2013 and November 2015 were analysed and compared to two different commercial drug specific calibrators from different manufacturers for each DOAC. Our results show that dabigatran (Hyphen and Technoclone) and rivaroxaban (Stago) calibrators tend to overestimate the APTT but are similar to patient samples for PT. A cut-off DOAC level of $50 \mathrm{ng} / \mathrm{mL}$ based on results from patient samples within the laboratory can be used as the lower limit which will result in prolongation of APTT for dabigatran (sensitivity $96 \%, n=25$ ) and PT for rivaroxaban (sensitivity $97 \%$, $n=29$ ), respectively. Individual laboratories should be familiar with the sensitivity of their coagulation reagents to different DOACs including differences between patient samples versus different commercial drug specific calibrators.
\end{abstract}

Key words: Direct oral anticoagulants; DOACs; activated partial thromboplastin time; prothrombin time; patient samples; calibrators.

Received 15 May, revised 13 July, accepted 18 July 2016

Available online 22 October 2016

\section{INTRODUCTION}

Direct oral anticoagulants (DOACs) have the advantage of more predictable pharmacokinetics and pharmacodynamics compared to vitamin $\mathrm{K}$ antagonists such as warfarin and therefore do not require routine laboratory monitoring. However in some situations, such as bleeding, recurrent or progressive thrombosis, emergency surgery, renal failure or liver failure, laboratory monitoring may be required. This includes baseline coagulation assays and functional anticoagulant levels. Previous studies have shown that the sensitivities of coagulation assays to the DOACs are reagent and method dependent. ${ }^{1-7}$ Most of these early studies have been based on spiked normal pooled plasma. Very few studies were performed using patient samples. ${ }^{8-12}$ Regarding the use of routine coagulation assays in the laboratory assessment of DOACs, most guidelines recommend the use of activated partial thromboplastin time (APTT) for dabigatran and prothrombin time (PT) for rivaroxaban for urgent screening of DOACs when the drug being assessed is known. ${ }^{13}$ Previous guidelines such as the 2012 British Committee for Standards in Haematology (BCSH) have suggested that a normal APTT for dabigatran and PT for rivaroxaban excludes a therapeutic intensity of the drug. ${ }^{14}$ This has been called into question by several later studies performed using patient samples which showed coagulation tests within the normal range even at therapeutic levels of DOAC. In addition, the use of calibrators (spiked samples) tended to overestimate the sensitivity of routine coagulation tests to dabigatran and rivaroxaban. ${ }^{10,15-19}$ In Australia, current guidelines by the Australasian Society of Thrombosis and Haemostasis (ASTH) recommend using specific quantitative assays, i.e., dilute thrombin time and drug specific anti-factor Xa chromogenic assay to assess dabigatran and rivaroxaban levels, respectively. ${ }^{20}$ Since these assays may not be available in many regional and remote laboratories and/or after hours, ASTH recognises that routine coagulation assays such as thrombin time (TT), APTT and PT can be utilised as screening tests to provide qualitative information about the presence of DOACs. However, caution should be given in adopting these recommendations if commercial drug-specific calibrators are used to determine the relative sensitivity of routine coagulation to DOACs, as there are differences in spiked compared to patient samples. Previous studies have comprehensively evaluated the effect of DOACs on haemostasis tests in spiked or patient samples but none have compared the sensitivity of both the APTT and PT to different DOACs in patient samples versus different drug-specific commercial calibrators. ${ }^{11,12,16}$ A recent study evaluated the use of a single drug-specific commercial calibrator for determining PT or APTT reagent sensitivity to dabigatran and rivaroxaban compared to patient 
samples but not for apixaban or using different drug-specific commercial calibrators. ${ }^{18}$

Actin FS and Neoplastine CI Plus are the most common APTT and PT reagents, respectively, used in Australian laboratories, with a third of laboratories using these reagents. In a survey of laboratories participating in the Royal College of Pathologists of Australasia Quality Assurance Programs (RCPA QAP) Haemostasis Program, Actin FS was the most commonly used APTT reagent in 194 of 708 laboratories (27\%) among 15 other reagents. Neoplastine CI Plus was the second most common PT reagent used after SM Thrombrel S in 240 out of 730 laboratories $(33 \%)$ among nine other PT reagents. ${ }^{21}$

Our first aim was to use a common APTT and PT reagent in Australia (Actin FS and Neoplastine CI Plus, respectively) to compare the sensitivities of routine coagulation assays to DOACs in patient samples versus different commercial drugspecific calibrators. If routine coagulation studies can be used as a rough guide to determine whether significant DOAC is present, it would also be useful to know what approximate level of DOAC would cause prolongation of the APTT or PT using these reagents. The median trough level for dabigatran, rivaroxaban and apixaban has been estimated to be around $90 \mathrm{ng} / \mathrm{mL}$ [95\% confidence interval (CI) 31-225], $32 \mathrm{ng} / \mathrm{mL}$ [interquartile range (IQR) $19-60$ ] and $63 \mathrm{ng} / \mathrm{mL}(95 \% \mathrm{CI}$ 22-177), respectively. ${ }^{22-24}$ Corresponding median peak levels were $184 \mathrm{ng} / \mathrm{mL}$ (95\% CI 64-443), $244 \mathrm{ng} / \mathrm{mL}$ (IQR 175-360) and $132 \mathrm{ng} / \mathrm{mL}$ (95\%CI 59-302), respectively. Given the lower level sensitivity of the quantitating assays (approximately $40 \mathrm{ng} / \mathrm{mL}$ for dilute thrombin time; $25 \mathrm{ng} / \mathrm{mL}$ for chromogenic anti Xa assays), ${ }^{25-29}$ a level of $50 \mathrm{ng} / \mathrm{mL}$ may be a reasonable target to suggest a limited haemostasis related effect at that concentration/time point. Some studies have reported so called 'peak' level ranges for some DOACs of below $50 \mathrm{ng} / \mathrm{mL},{ }^{13,22,30,31}$ although it would be expected that this will be a low occurrence rate, and potentially representative of 'non-compliance' or maybe even 'DOAC resistance' in occasional patients. Therefore, the second aim of this study was to determine the distribution of samples with routine coagulation assays above the normal range at a specified cut-off drug level of $50 \mathrm{ng} / \mathrm{mL}$ using different DOACs in patient samples.

\section{MATERIALS AND METHODS}

\section{Patient samples}

For this analysis, patient samples collected at Pathology North Hunter (PN-H) between February 2012 and November 2015 requesting for dabigatran $(n=65)$, rivaroxaban $(n=112)$, or apixaban levels $(n=31)$ were analysed retrospectively. The data analysed consisted of results from coagulation assays performed using Neoplastine CI Plus (Diagnostica Stago, France) and Actin FS (Siemens, Germany) which are the primary reagents used in our laboratory for PT and APTT testing, respectively. The testing was done by mechanical clot detection method on a STA-R Evolution instrument (Diagnostica Stago). Dabigatran levels were tested by an in-house dilute thrombin time akin to the commercially available Hemoclot assay from Hyphen Biomed. Rivaroxaban and apixaban levels were performed using Liquid antiXa chromogenic assay (STA liquid anti-Xa; Diagnostica Stago). Limits of detection for dilute thrombin time and liquid anti-Xa chromogenic assays are $40 \mathrm{ng} / \mathrm{mL}^{26}$ and $25 \mathrm{ng} / \mathrm{mL}$ (package insert from manufacturer), respectively. Levels below this were reported as 'zero'. Patient samples where neither APTT nor PT were requested (dabigatran $n=9$; rivaroxaban $n=41$; apixaban $n=8$ ) were excluded. Patient samples where only one of the PT or APTT results were available which made up the minority were included in analysis for the individual tests. Further samples were excluded if patients were on other oral anticoagulants $(n=3)$, had liver disease $(n=7)$, multiorgan failure $(n=1)$ or if coagulation tests were abnormal when levels of DOACs were undetectable $(n=18)$. After exclusions, the number of remaining samples available for the final analysis were dabigatran $(n=39)$, rivaroxaban $(n=56)$, and apixaban $(n=22)$.

\section{Commercial drug-specific calibrators}

PT and APTT were performed on commercial drug-specific calibrators in which DOAC levels were predetermined by the manufacturer using liquid chromatography mass spectrometry (LC/MS-MS). Two calibrators sourced from different manufacturers were used for each DOAC. For rivaroxaban and apixaban, the calibrators were sourced from Diagnostica Stago (France) and Technoclone (Austria), while for Dabigatran the calibrators were sourced from Hyphen Biomed (France) and Technoclone (Austria).

\section{Data analysis}

Data were analysed using Microsoft Excel (Microsoft, USA). Linear regression lines and correlation coefficient values, $\mathrm{R}^{2}$ were derived using the statistical software package included in Microsoft Excel. To compare the sensitivity of APTT and PT in patient samples with commercial drug-specific calibrators, APTT and PT were plotted against DOAC level in separate graphs for each DOAC.

To further evaluate the differences between patient samples and commercial drug-specific calibrators, we used difference plots (Bland-Altman plots) obtained from the Analyse-it extension statistical software package for Excel. For this analysis, a derived PT or APTT for the corresponding DOAC level in patient samples was obtained from the drug-specific calibrator curve. The difference or percentage difference between the derived APTT/PT from the calibrator curve and the APTT/PT for patient samples was then plotted against the mean APTT/PT (mean of APTT/PT from patient samples and derived APTT/PT from calibrator curve). Mean and mean percentage differences were indicated by the 'plus' sign if the derived clotting time was more prolonged and 'minus' sign if the clotting time was shorter for commercial drug-specific calibrators compared to patient samples. A two-tailed paired $t$ test was used to determine if the difference between mean APTT/PT for patient samples compared with mean derived APTT/PT for commercial drug-specific calibrators were significant $(p<0.05)$.

\section{Ethics}

This study was approved by Hunter New England (NSW) Human Research Ethics Committee.

\section{RESULTS}

\section{Sensitivity of APTT and PT to DOACs in patient samples compared with commercial drug-specific calibrators}

The DOAC levels used for commercial drug-specific calibrators from various manufacturers and their corresponding APTT/PT values are summarised in Table 1. Difference results from the Bland-Altman analysis comparing sensitivity of APTT and PT to DOACs in patient samples versus drugspecific commercial calibrators are shown in Table 2. For DOAC levels in patient samples, a level below the limit of detection in our laboratory as described in methods was reported as 'zero'.

\section{Dabigatran}

Dabigatran levels in patient samples ranged from 0 to $667 \mathrm{ng} /$ $\mathrm{mL}$ and the corresponding APTT and PT ranges were 28-104 s and 13-33 s, respectively. Correlation between APTT clotting times and dabigatran levels in commercial drug-specific calibrators $\left(R^{2}=1\right.$ for Hyphen and $R^{2}=0.9959$ for Technoclone) was higher compared to patient samples $\left(\mathrm{R}^{2}=0.7717\right)$. Drug-specific calibrators overestimated the sensitivity of the APTT to dabigatran compared to patient 
Table 1 Summary of DOAC levels used for spiked samples using calibrators from various manufacturers and their corresponding APTT/PT values

\begin{tabular}{|c|c|c|c|c|c|c|c|c|c|c|c|}
\hline \multicolumn{4}{|c|}{ Dabigatran } & \multicolumn{4}{|c|}{ Rivaroxaban } & \multicolumn{4}{|c|}{ Apixaban } \\
\hline Calibrator & Level (ng/mL) & APTT, s & PT, s & Calibrator & Level (ng/mL) & APTT, s & PT, s & Calibrator & Level (ng/mL) & APTT, s & $\mathrm{PT}, \mathrm{s}$ \\
\hline \multirow[t]{4}{*}{ Hyphen } & 30 & 49.9 & 17.0 & Stago & 0 & 33.1 & 13.4 & Stago & 0 & 29.8 & 14.2 \\
\hline & 255 & 84 & 23.8 & & 98 & 42.8 & 19.2 & & 90 & 31.5 & 15.3 \\
\hline & 468 & 106 & 36.2 & & 241 & 52.9 & 26.5 & & 228 & 32.9 & 16.8 \\
\hline & & & & & 495 & 66.4 & 36.6 & & 455 & 36.2 & 19.6 \\
\hline \multirow{4}{*}{ Tech } & 65.2 & 48.1 & 15.7 & & 48.3 & 35.0 & 15.5 & & 64.4 & 34.3 & 14.3 \\
\hline & 212.7 & 64.7 & 19.3 & & 101.3 & 41.9 & 19.8 & & 215.9 & 36.1 & 16.0 \\
\hline & 408.6 & 84.4 & 24.4 & & 199.2 & 48.1 & 23.9 & & 433 & 37.7 & 17.5 \\
\hline & 514.8 & 93.2 & 29.3 & & 433.3 & 55.4 & 34.3 & & 623.5 & 39.7 & 19.1 \\
\hline
\end{tabular}

APTT, activated partial thromboplastin time; Hyphen, Hyphen Biomed; PT, prothrombin time; s, seconds; Tech, Technoclone.

Table 2 Summary of Bland-Altman analysis

\begin{tabular}{|c|c|c|c|c|}
\hline \multirow[t]{2}{*}{ DOAC in spiked samples } & \multicolumn{2}{|c|}{$\begin{array}{c}\text { APTT bias (derived APTT from spiked sensitivity curve - } \\
\text { APTT patient samples) }\end{array}$} & \multicolumn{2}{|c|}{$\begin{array}{c}\text { PT bias (derived PT from spiked sensitivity curve - } \\
\text { PT patient samples) }\end{array}$} \\
\hline & $\begin{array}{l}\text { Mean difference, } \\
\text { s }(95 \% \mathrm{CI})\end{array}$ & $\begin{array}{c}\text { Mean percentage } \\
\text { difference, \% }(95 \% \mathrm{CI})\end{array}$ & $\begin{array}{l}\text { Mean difference, } \\
\text { s }(95 \% \mathrm{CI})\end{array}$ & $\begin{array}{c}\text { Mean percentage } \\
\text { difference, } \%(95 \% \mathrm{CI})\end{array}$ \\
\hline $\begin{array}{l}\text { Dabigatran } \\
\text { (Hyphen) }\end{array}$ & $+16.4(-2.9,35.8)^{\mathrm{a}}$ & $+29.9(0.3,59.5)^{\mathrm{a}}$ & $+2.2(-4.6,9.0)^{\mathrm{a}}$ & $+10.4(-13.7,34.4)^{\mathrm{a}}$ \\
\hline $\begin{array}{l}\text { Dabigatran } \\
\text { (Technoclone) }\end{array}$ & $+4.5(-12.9,21.9)^{\mathrm{a}}$ & $+9.3(-21.3,39.9)^{\mathrm{a}}$ & $-0.9(-4.4,2.6)^{\mathrm{a}}$ & $-4.8(-25.1,15.4)^{\mathrm{a}}$ \\
\hline $\begin{array}{l}\text { Rivaroxaban } \\
\text { (Stago) }\end{array}$ & $+4.0(-6.6,14.6)^{\mathrm{a}}$ & $+10.2(-18.3,38.7)^{\mathrm{a}}$ & $-0.5(-4.7,3.6) \mathrm{NS}$ & $-2.6(-21.3,16.2) \mathrm{NS}$ \\
\hline $\begin{array}{l}\text { Rivaroxaban } \\
\text { (Technoclone) }\end{array}$ & $+1.4(-9.6,12.4) \mathrm{NS}$ & $+3.4(-27.6,34.4) \mathrm{NS}$ & $-0.6(-4.8,3.6)^{\mathrm{b}}$ & $-3.0(-22.0,15.9)^{\mathrm{b}}$ \\
\hline $\begin{array}{l}\text { Apixaban } \\
\text { (Stago) }\end{array}$ & $-0.5(-8.3,7.4) \mathrm{NS}$ & $-0.5(-24.2,23.1) \mathrm{NS}$ & $-1.0(-3.8,1.8)^{\mathrm{a}}$ & $-5.8(-21.7,10.1)^{\mathrm{a}}$ \\
\hline $\begin{array}{l}\text { Apixaban } \\
\text { (Technoclone) }\end{array}$ & $+2.4(-5.6,10.5)^{\mathrm{b}}$ & $+8.4(-24.6,33.0)^{\mathrm{a}}$ & $-1.6(-4.7,1.5)^{\mathrm{a}}$ & $-9.9(-27.0,7.2)^{\mathrm{a}}$ \\
\hline
\end{tabular}

Shown are the activated partial thromboplastin time (APTT)/prothrombin time (PT) bias for each DOAC expressed as mean difference or mean percentage difference. $95 \%$ confidence intervals $(\mathrm{CI})$ for mean difference or mean percentage difference are indicated in parentheses.

Corresponding $p$ values for mean difference/mean percentage difference were obtained using a two-tailed paired $t$ test: ${ }^{\mathrm{a}} p<0.01 ;{ }^{\mathrm{b}} p<0.05$; NS, not significant, i.e., $p>0.05$.

samples (Fig. 1A). The mean difference in APTT was $+16.4 \mathrm{~s}$ (95\% CI -2.9, 35.8 s) using Hyphen Biomed calibrators and +4.5 s $(95 \% \mathrm{CI}-12.9,21.9 \mathrm{~s})$ using Technoclone calibrators by Bland-Altman analysis. Correlation between PT clotting times and dabigatran levels in commercial drugspecific calibrators $\left(\mathrm{R}^{2}=1\right.$ for Hyphen and $\mathrm{R}^{2}=0.9948$ for Technoclone) was also higher compared to patient samples $\left(\mathrm{R}^{2}=0.8457\right)$. Sensitivity of PT to dabigatran was similar in patient samples and Technoclone calibrators with a mean difference of $-0.9 \mathrm{~s}(95 \% \mathrm{CI}-4.4,2.6 \mathrm{~s})$ (Fig. 1B). At higher concentrations of dabigatran, PT was increasingly more sensitive in Hyphen Biomed calibrators compared to patient samples with a mean difference $+2.2 \mathrm{~s}(95 \% \mathrm{CI}-4.6,9.0 \mathrm{~s})$.

\section{Rivaroxaban}

Rivaroxaban levels in patient samples ranged from 0 to $381 \mathrm{ng} / \mathrm{mL}$. The corresponding APTT and PT ranges were 19-55 s and 14-38 s, respectively. There was better correlation between APTT clotting times and rivaroxaban levels in commercial drug-specific calibrators $\left(\mathrm{R}^{2}=0.9993\right.$ for Stago and $\mathrm{R}^{2}=0.9963$ for Technoclone) compared to patient samples $\left(R^{2}=0.5397\right)$. Stago calibrators overestimated the
APTT compared to the patient samples with mean difference of $+4.0 \mathrm{~s}(95 \% \mathrm{CI}-6.6,14.6 \mathrm{~s})$ whereas Technoclone calibrators had similar APTT sensitivity to patient samples, i.e., +1.4 s (95\% CI -9.6, 12.4 s) (Fig. 1C). Correlation between PT clotting times and commercial drug-specific commercial calibrators $\left(R^{2}=0.9992\right.$ for Stago and $R^{2}=0.9930$ for Technoclone) was higher compared to patient samples $\left(\mathrm{R}^{2}=0.8518\right)$. Sensitivity of PT to rivaroxaban was similar in Stago and Technoclone calibrators compared to patient samples with mean differences of $-0.5 \mathrm{~s}(95 \% \mathrm{CI}-4.7,3.6 \mathrm{~s})$ and $-0.6 \mathrm{~s}(95 \% \mathrm{CI}-4.8,3.6 \mathrm{~s})$, respectively (Fig. 1D).

\section{Apixaban}

Apixaban levels in patient samples ranged from 0 to $283 \mathrm{ng} /$ $\mathrm{mL}$. The corresponding APTT and PT ranges were 26-41 s and 14-22 s, respectively. There was poor correlation between APTT clotting times and apixaban levels in patient samples $\left(\mathrm{R}^{2}=0.4811\right)$ but good correlation of APTT clotting times with apixaban levels in commercial drug-specific calibrators $\left(\mathrm{R}^{2}=0.9931\right.$ for Stago and $\mathrm{R}^{2}=0.9601$ for Technoclone). Correlation between PT clotting times and apixaban levels in commercial drug-specific calibrators 

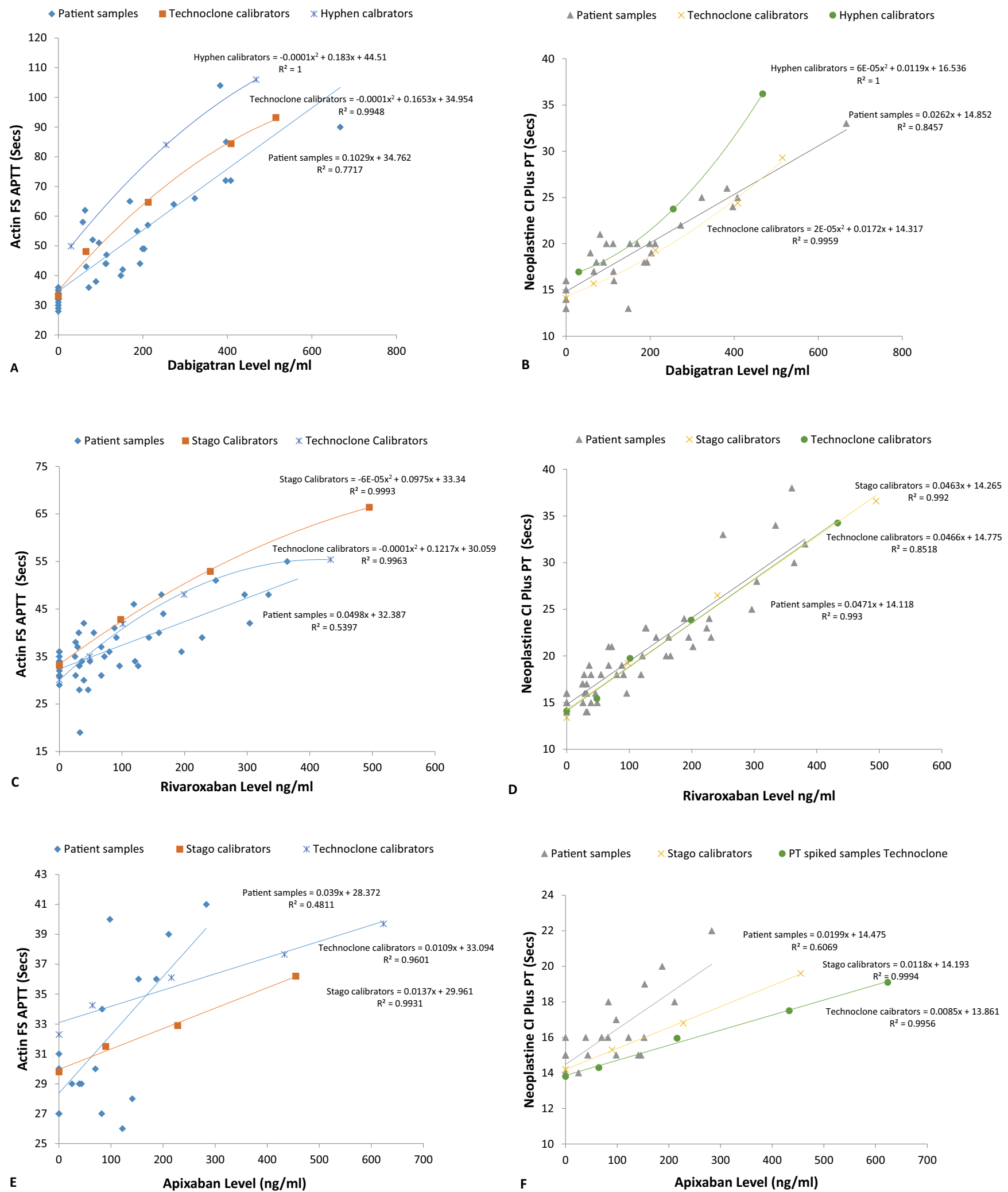

Fig. 1 Sensitivity of APTT Actin FS and PT Neoplastine CI Plus to DOACs in patient samples vs commercial drug-specific calibrators. (A) Dabigatran: APTT in patient samples versus Technoclone or Hyphen Biomed calibrators. (B) Dabigatran: PT in patient samples versus Technoclone or Hyphen Biomed calibrators. (C) Rivaroxaban: APTT in patient samples versus Stago or Technoclone calibrators. (D) Rivaroxaban: PT in patient samples versus Stago or Technoclone calibrators. (E) Apixaban: APTT in patient samples versus Stago or Technoclone calibrators. (F) Apixaban: PT in patient samples versus Stago or Technoclone calibrators. Shown also are the equations and correlation coefficients $\left(\mathrm{R}^{2}\right)$ for the line of best fit. DOAC levels below the limits of detection $(<40 \mathrm{ng} / \mathrm{mL}$ for dabigatran, $<25 \mathrm{ng} / \mathrm{mL}$ for rivaroxaban and apixaban) in our laboratory were reported as 'zero'. 
$\left(\mathrm{R}^{2}=0.9994\right.$ for Stago and $\mathrm{R}^{2}=0.9956$ for Technoclone) was higher compared to patient samples $\left(\mathrm{R}^{2}=0.6069\right)$. In contrast to dabigatran and rivaroxaban, both APTT at higher concentrations (Fig. 1E) and PT (Fig. 1F) appeared to be less sensitive to apixaban in commercial drug-specific calibrators compared to patient samples.

Effect of DOACs on routine coagulation assays above and below a specified cut-off drug level of $>50 \mathrm{ng} / \mathrm{mL}$ using Neoplastine CI Plus and Actin FS as reagents in patient samples

APTT above and below a cut-off DOAC level of $50 \mathrm{ng} / \mathrm{mL}$

The APTT was prolonged with dabigatran levels above 50 $\mathrm{ng} / \mathrm{mL}$ (median $169 \mathrm{ng} / \mathrm{mL}$ IQR 96-273 ng/mL) in all samples apart from one sample which had an APTT at the upper limit of normal range, i.e., $36 \mathrm{~s}$ [APTT reference range (RR) 24-36 s] with a dabigatran level of $72 \mathrm{ng} / \mathrm{mL}$. APTT was not prolonged in seven of 22 samples at rivaroxaban levels above $50 \mathrm{ng} / \mathrm{mL}$ (median $135 \mathrm{ng} / \mathrm{mL}$, IQR 89-220 ng/ $\mathrm{mL}$ ). Seven of 10 samples were within the normal range for APTT at apixaban levels of above $50 \mathrm{ng} / \mathrm{mL}$ (median $132 \mathrm{ng}$ / $\mathrm{mL}$, IQR 87-179 ng/mL) (Fig. 2A).

PT above and below a cut-off DOAC level of $50 \mathrm{ng} / \mathrm{mL}$

PT was sensitive to dabigatran at levels above $50 \mathrm{ng} / \mathrm{mL}$ (median $169 \mathrm{ng} / \mathrm{mL}$, IQR 104-243 ng/mL) for all but two samples, which yielded results within the normal range (i.e., PT $13 \mathrm{~s}$ at dabigatran level 148 and PT $16 \mathrm{~s}$ at dabigatran level 114). For rivaroxaban, the PT was sensitive to
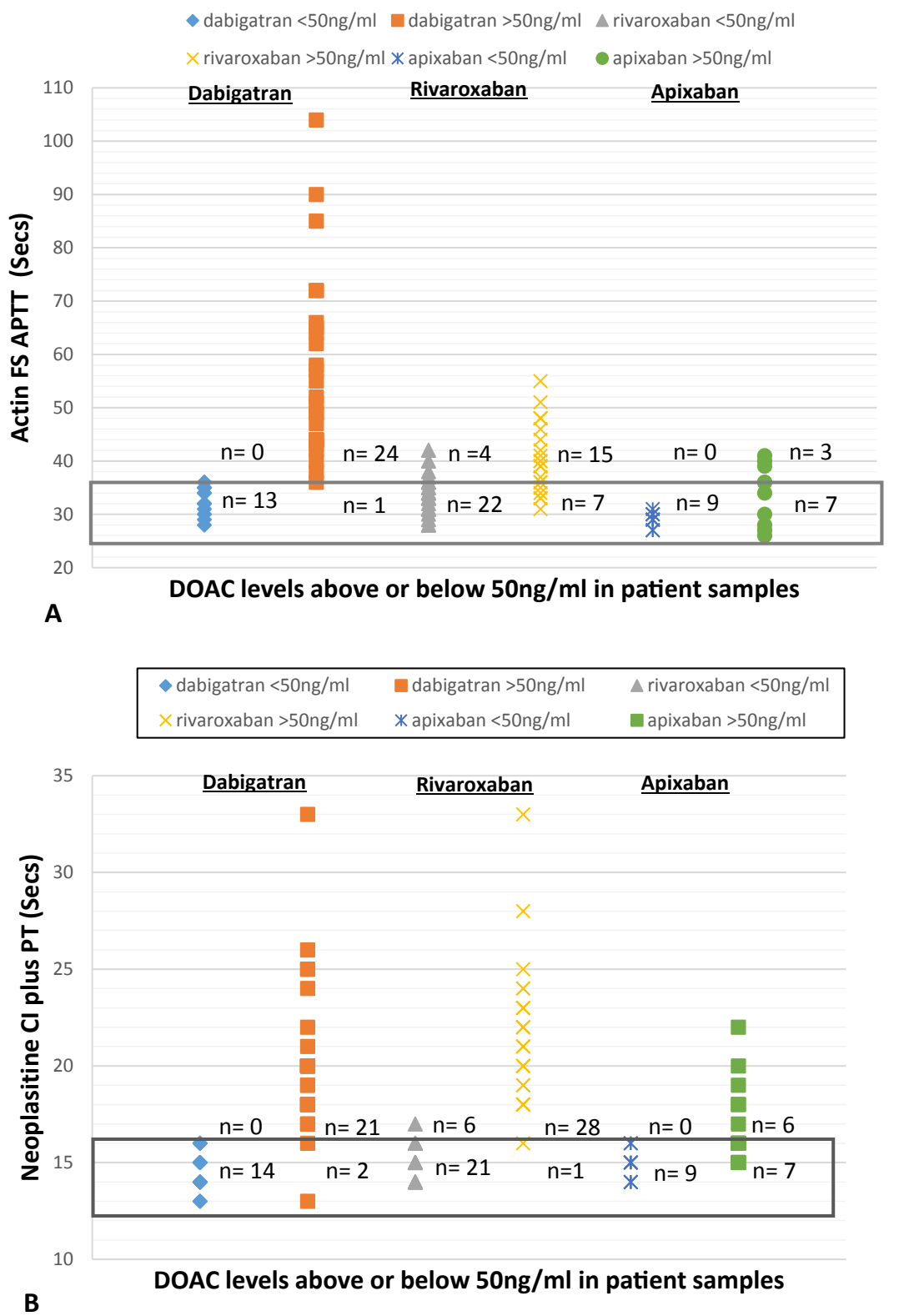

Fig. 2 Distribution of samples with prolonged coagulation tests above and below a cut-off drug level of $50 \mathrm{ng} / \mathrm{mL}$ for (A) APTT and (B) PT. Boxed area indicates the normal range for the specific coagulation assay, i.e., 24-36 s for APTT and 12-16 s for PT. $\mathrm{n}=$ number of samples within or above the normal range for the APTT or PT. 
rivaroxaban levels above $50 \mathrm{ng} / \mathrm{mL}$ (median $163 \mathrm{ng} / \mathrm{mL}, \mathrm{IQR}$ 96-231 ng/mL). Only one of 29 samples did not have a prolonged PT (PT $16 \mathrm{~s}$, RR 12-16 s) at rivaroxaban level $98 \mathrm{ng} / \mathrm{mL}$. Apixaban was not sensitive to PT at levels above $50 \mathrm{ng} / \mathrm{mL}$ (median $141 \mathrm{ng} / \mathrm{mL}$, IQR $98-153 \mathrm{ng} / \mathrm{mL}$ ) with seven of 13 samples within the normal range (Fig. 2B).

\section{DISCUSSION}

\section{Differences between patient and commercial drug- specific calibrators}

Commercial drug-specific calibrators and patient samples differ in several ways. Spiked normal pooled plasma is readily available from various manufacturers as commercial calibrators for coagulation assays and these are preferred in laboratory quality control programs as well as research studies. In contrast, patient samples requires recruitment of individual donors which is challenging and time consuming. Commercial calibrators are usually lyophilised (freeze dried) prior to storage and have to be reconstituted. Studies have shown that clotting factor activities in lyophilised plasma remain stable after 24 months of storage at room temperature and up to 6 days after reconstitution when stored at $4^{\circ} \mathrm{C}^{32,33}$ Based on these studies, loss of clotting factor activities due to prolonged storage and reconstitution in lyophilised plasma would not be anticipated to have a significant effect on routine coagulation assays. However, there are some studies that have shown that the use of lyophilised plasma may prolong PT and APTT values compared to fresh plasma. ${ }^{34,35}$ This suggests that these differences may be due to intrinsic differences in factor concentrations at source which may vary depending on the manufacturer or lot number of the particular plasma used. As drug-specific commercial calibrators used to quantitate DOAC levels are only designed to measure the drug level, the 'normality' of the plasma spiked with the DOAC is not usually considered important by the manufacturer as each DOAC assay is designed to be insensitive to any coexisting factor deficiencies. Thus spiked 'normal' pooled plasma used in commercial drug-specific calibrators in fact may be variable in regards to concentration of some clotting factors. In keeping with this, only drug levels, and not the factor levels, in commercial drug-specific calibrators used for coagulation assays are routinely checked prior to release by some manufacturers (personal correspondence via email).

\section{Sensitivity of routine coagulation assays to DOACs in patient samples versus commercial drug-specific calibrators}

Previous studies have shown that the APTT is more sensitive to dabigatran and rivaroxaban in patient samples compared with spiked samples. ${ }^{4}$ To our knowledge, this is the first study to compare the sensitivity of both APTT and PT to different DOACs in patient samples versus different drugspecific commercial calibrators. Our study shows that differences in sensitivity of the coagulation assays in patient samples compared to drug-specific commercial calibrators vary depending on (1) type of coagulation assay, (2) DOAC used, and (3) the commercial drug-specific calibrator used to establish the spiked DOAC sensitivity curves. Reasons for the differences in sensitivity to DOACs in calibrator material compared to patient samples are unclear, although one study suggested that this may be due to antibiotic used as a preservative in calibrator material affecting some PT and APTT reagents. ${ }^{18}$ Recommendations on the use of PT and APTT alone as an indicator of DOAC levels based on commercial drug-specific calibrators therefore should be interpreted with caution as routine coagulation assays are based on patient samples. For example, a normal APTT in patient samples for dabigatran and rivaroxaban may correspond to a higher level of DOAC than suggested by studies based on drug-specific commercial calibrators as the APTT is less sensitive in patient samples. However, whether these differences translate clinically into significant differences in bleeding is unknown.

\section{Using cut-offs to estimate DOAC levels based on routine coagulation tests in patient samples}

Using Actin FS and Neoplastine CI Plus as reagents in patient samples, a specified cut-off drug concentration of $50 \mathrm{ng} / \mathrm{mL}$ could be used as an estimate of the lower limit above which the APTT for dabigatran and the PT for rivaroxaban would be prolonged in most samples. To our knowledge, our study is the first to report this. This was not the case for apixaban which had no effect on the APTT and $\mathrm{PT}$, even at concentrations exceeding $100 \mathrm{ng} / \mathrm{mL}$. In general, a DOAC concentration of $<50 \mathrm{ng} / \mathrm{mL}$ would suggest a limited haemostasis related effect at that concentration/time point. Thus a normal APTT for dabigatran and PT for rivaroxaban can be used as a rough guide as to whether significant drug is present using a sensitive reagent such as Actin FS for APTT and Neoplastine CI Plus for PT. This would be especially useful for laboratories where routine or after hours drug levels are not available. However for accurate estimation, the dilute thrombin time for dabigatran and drug specific anti-factor Xa assay for rivaroxaban and apixaban should still be used. ${ }^{14,20,36-38}$ Indeed, most recent guidelines accept that a normal PT and APTT will not exclude possible rivaroxaban and dabigatran 'within therapy range', although this will be true for most samples giving normal PT and APTT. ${ }^{15,39-41}$ It is also important to emphasise that these results may vary if a different reagent/ instrument combination is used. ${ }^{15}$ Hence each laboratory should be aware of the sensitivity of their coagulation assays to the DOACs.

\section{Comparison and correlation of this study with previously published studies}

The British Committee for Standards in Haematology (BCSH), as well as the Subcommittee on Control of Anticoagulation of the Scientific and Standardization Committee of the International Society on Thrombosis and Haemostasis (ISTH) state that the APTT and PT can be used to determine the relative intensity of anticoagulation caused by dabigatran and rivaroxaban respectively if drug specific quantitative assays are not available. ${ }^{14,36}$ In addition, the $2012 \mathrm{BCSH}$ guidelines propose that that a normal APTT for dabigatran and PT for rivaroxaban excludes a therapeutic intensity of the drug. ${ }^{14}$ These international recommendations suggest that each laboratory should be aware of the sensitivity of their own PT and APTT assays to dabigatran and rivaroxaban which can be achieved using commercially available dabigatran and rivaroxaban calibrators. A recently published study by Gosselin et al. ${ }^{18}$ compared the use of a single drug- 
specific commercial calibrator to patient samples for determining PT or APTT reagent sensitivity to dabigatran and rivaroxaban. They demonstrated significant differences and cautioned against the use of drug-specific calibrators to determine the effect of DOACs on routine coagulation tests and other specialised haemostasis assays (e.g., factor levels and protein S). They also compared the sensitivity of three different APTT and PT reagents to dabigatran and rivaroxaban using Hyphen Biomed calibrators and patient samples. APTT reagents used, i.e., SynthASil, Actin FSL, and PTT-A were different from the APTT reagent used in our study (Actin FS). Of the three PT reagents used, i.e., Recombiplastin 2G, Innovin, and Neoplastine CI plus, one was similar to that used in our study (Neoplastine CI plus). Relative sensitivities were compared using drug concentration required to double the clotting time (2CT) from baseline of the normal pooled plasma (NPP) sample as opposed to the Bland-Altman method that we used in our study. It was shown that Hyphen calibrators overestimated APTT or PT sensitivity to dabigatran compared to patient samples using Actin FSL and all three PT reagents. In our study using Actin FS as APTT reagent, both Hyphen and Technoclone calibrators also overestimated APTT sensitivity to dabigatran compared to patient samples. Using Neoplastine CI Plus as PT reagent, Hyphen calibrators overestimated the sensitivity of the PT to dabigatran similar to the above study but Technoclone calibrators underestimated the PT (Table 2). This suggests that the relative sensitivity of routine coagulation assays to dabigatran in drug-specific calibrators compared with patient samples are both reagent and calibrator specific. With regards to rivaroxaban, Gosselin et al. ${ }^{18}$ demonstrated that Hyphen calibrators overestimated the sensitivity of all APTT and PT reagents used in their study compared to patient samples including the PT reagent Neoplastine CI Plus as used in our study. In our study, different calibrators (Stago and Technoclone) were used but using the same PT reagent (Neoplastine CI Plus) the sensitivity of PT to rivaroxaban in Stago and Technoclone calibrators were similar to patient samples. The reasons for differences in the relative sensitivities of APTT and PT to dabigatran and rivaroxaban in drug-specific calibrators compared with patient samples using different reagentcalibrator combinations observed in our study and the study by Gosselin et al. are unclear. However, it should be noted that different methods were used for comparing relative sensitivities. It is also interesting to note that using one calibrator and reagent combination similar in both studies, i.e., Hyphen and Neoplastine CI Plus, the relationship between dabigatran levels and PT in our study (curvilinear) was different compared to that obtained by Gosselin et al. (linear). ${ }^{18}$

\section{Study limitations}

Limitations of our study include the limited number of reagents and commercial drug-specific calibrators used. Future studies comparing the sensitivity of routine coagulation assays in patient samples versus commercial drugspecific calibrators using different reagent and commercial drug-specific calibrator combinations would be informative. In addition, being a retrospective study, a number of samples ( $n=26$ dabigatran, $n=56$ rivaroxaban and $n=9$ apixaban) had to be excluded because of missing information.

\section{CONCLUSION}

Each laboratory has to be familiar with the sensitivity of their coagulation assays to different DOACs including differences in patient samples compared to different commercial drugspecific calibrators. Using a sensitive reagent, a cut-off DOAC level based on patient samples which will result in prolongation of APTT for dabigatran and PT for rivaroxaban can be established in individual laboratories and used as a rough guide for whether significant DOAC is present when specific assays for DOAC levels are not available.

Acknowledgements: The authors thank Conjoint A/Prof Lisa F. Lincz for her help in improvisation and revision of the manuscript and Priscilla Swanepoel for performing the assays and providing technical assistance.

Conflicts of interest and sources of funding: The Royal College of Pathologists of Australasia Specialist Training Program (RCPA STP) Rural Supplementary Funds and Pathology North Hunter (PN-H) Trust Funds kindly provided funding for this project. The authors state there are no conflicts of interest to disclose.

Address for correspondence: Dr Ming Sheng Lim, Haematology Department, Calvary Mater Hospital, Level 4 New Med. Calvary Mater Hospital Edith St, Waratah, NSW 2298, Australia. E-mail: mingsheng.lim@ calvarymater.org.au

\section{References}

1. Hillarp A, Baghaei F, Fagerberg Blixter I, et al. Effects of the oral, direct factor Xa inhibitor rivaroxaban on commonly used coagulation assays. J Thromb Haemost 2011; 9: 133-9.

2. Douxfils J, Mullier F, Robert S, et al. Impact of dabigatran on a large panel of routine or specific coagulation assays: laboratory recommendations for monitoring of dabigatran etexilate. Thromb Haemost 2012 107: 985-97.

3. Samama MM, Guinet C, Le Flem L, et al. Measurement of dabigatran and rivaroxaban in primary prevention of venous thromboembolism in 106 patients, who have undergone major orthopedic surgery: an observational study. J Thromb Thrombolysis 2013: 35: 140-6.

4. Helin TA, Pakkanen A, Lassila R, et al. Laboratory assessment of novel oral anticoagulants: method suitability and variability between coagulation laboratories. Clin Chem 2013; 59: 807-14.

5. Van Blerk M, Bailleul E, Chatelain B, et al. Influence of dabigatran and rivaroxaban on routine coagulation assays. A nationwide Belgian survey. Thromb Haemost 2015; 113: 154-64.

6. Lindahl TL, Baghaei F, Blixter IF, et al. Effects of the oral, direct thrombin inhibitor dabigatran on five common coagulation assays. Thromb Haemost 2011; 105: 371-8.

7. Douxfils J, Mullier F, Loosen C, et al. Assessment of the impact of rivaroxaban on coagulation assays: laboratory recommendations for the monitoring of rivaroxaban and review of the literature. Thromb Res 2012; 130: 956-66.

8. Liesenfeld K-H, Schäfer HG, Trocóniz IF et al. Effects of the direct thrombin inhibitor dabigatran on ex vivo coagulation time in orthopaedic surgery patients: a population model analysis. Br J Clin Pharmacol 2006; 62: 527-37.

9. Asmis LM, Alberio L, Angelillo-Scherrer A, et al. Rivaroxaban: quantification by anti-FXa assay and influence on coagulation tests: a study in 9 Swiss laboratories. Thromb Res 2012; 129: 492-8.

10. Francart SJ, Hawes EM, Deal AM, et al. Performance of coagulation tests in patients on therapeutic doses of rivaroxaban. A cross-sectional pharmacodynamic study based on peak and trough plasma levels. Thromb Haemost 2014; 111: 1133-40.

11. Bonar R, Favaloro EJ, Mohammed S, Pasalic L, Sioufi J, Marsden K. The effect of dabigatran on haemostasis tests: a comprehensive assessment using in vitro and ex vivo samples. Pathology 2015; 47 $355-64$ 
12. Bonar R, Favaloro EJ, Mohammed S, et al. The effect of the direct factor Xa inhibitors apixaban and rivaroxaban on haemostasis tests: a comprehensive assessment using in vitro and ex vivo samples. Pathology 2016; 48: 60-71.

13. Lippi G, Favaloro EJ. Recent guidelines and recommendations for laboratory assessment of the direct oral anticoagulants (DOACs): is there consensus? Clin Chem Lab Med 2015; 53: 185-97.

14. Baglin T, Keeling D, Kitchen S. Effects on routine coagulation screens and assessment of anticoagulant intensity in patients taking oral dabigatran or rivaroxaban: guidance from the British Committee for Standards in Haematology. Br J Haematol 2012; 159: 427-9.

15. Cuker A, Siegal DM, Crowther MA, et al. Laboratory measurement of the anticoagulant activity of the non-vitamin $\mathrm{K}$ oral anticoagulants. J Am Coll Cardiol 2014; 64: 1128-39.

16. Adcock DM, Gosselin R. Direct oral anticoagulants (DOACs) in the laboratory: 2015 review. Thromb Res 2015; 136: 7-12.

17. Hawes EM, Deal AM, Funk-Adcock D, et al. Performance of coagulation tests in patients on therapeutic doses of dabigatran: a crosssectional pharmacodynamic study based on peak and trough plasma levels. J Thromb Haemost 2013; 11: 1493-502.

18. Gosselin RC, Adcock D, Hawes EM, et al. Evaluating the use of commercial drug-specific calibrators for determining PT and APTT reagent sensitivity to dabigatran and rivaroxaban. Thromb Haemost 2015 113: $77-84$

19. Gosselin R, Adcock DM. The laboratory's 2015 perspective on DOAC testing. J Thromb Haemost 2016; 13: 1-8.

20. Tran H, Joseph J, Young L, et al. New oral anticoagulants: a practical guide on prescription, laboratory testing and peri-procedural/bleeding management. Australasian Society of Thrombosis and Haemostasis. Intern Med J 2014; 44: 525-36.

21. The Royal College of Pathologists of Australasia Quality Assurance Programs (RCPA QAP), Haemostasis Program. Interim Report Cycle 12, Run 8. St Leonards: RCPA QAP, 2014.

22. van Ryn J, Stangier J, Haertter S, et al. Dabigatran etexilate - a novel, reversible, oral direct thrombin inhibitor: Interpretation of coagulation assays and reversal of anticoagulant activity. Thromb Haemost 2010 103: $1116-27$.

23. Buller HR, Lensing AWA, Prins $\mathrm{MH}$, et al. A dose-ranging study evaluating once-daily oral administration of the factor Xa inhibitor rivaroxaban in the treatment of patients with acute symptomatic deep vein thrombosis: the Einstein-DVT Dose-Ranging Study. Blood 2008; 112: $2242-7$.

24. Bristol-Myers Squib Canada. Eliquis (apixaban) Product Monograph. 11 Aug 2015; cited 12 Feb 2016. http://www.pfizer.ca/sites/g/files/ g10028126/f/201511/ELIQUIS_184490_mkt_PM_E.pdf

25. Schmitz EMH, Boonen K, van den Heuvel DJA, et al. Determination of dabigatran, rivaroxaban and apixaban by ultra-performance liquid chromatography - tandem mass spectrometry (UPLC-MS/MS) and coagulation assays for therapy monitoring of novel direct oral anticoagulants. J Thromb Haemost 2014; 12: 1636-46.

26. Douxfils J, Dogné J-M, Mullier F, et al. Comparison of calibrated dilute thrombin time and aPTT tests with LC-MS/MS for the therapeutic monitoring of patients treated with dabigatran etexilate. Thromb Haemost 2013; 110: 543-9.
27. Douxfils J, Tamigniau A, Chatelain B, et al. Comparison of calibrated chromogenic anti-Xa assay and PT tests with LC-MS/MS for the therapeutic monitoring of patients treated with rivaroxaban. Thromb Haemost 2013; 110: 723-31.

28. Samama MM, Contant G, Spiro TE, et al. Evaluation of the anti-factor $\mathrm{Xa}$ chromogenic assay for the measurement of rivaroxaban plasma concentrations using calibrators and controls. Thromb Haemost 2012; 107: 379-87.

29. Barrett YC, Wang Z, Frost C et al. Clinical laboratory measurement of direct factor Xa inhibitors: anti-Xa assay is preferable to prothrombin time assay. Thromb Haemost 2010; 104: 1263-71.

30. Mueck W, Lensing AWA, Agnelli G, et al. Rivaroxaban: population pharmacokinetic analyses in patients treated for acute deep-vein thrombosis and exposure simulations in patients with atrial fibrillation treated for stroke prevention. Clin Pharmacokinet 2011; 50: 675-86.

31. Becker RC, Alexander JH, Newby LK, et al. Effect of apixaban, an oral and direct factor $\mathrm{Xa}$ inhibitor, on coagulation activity biomarkers following acute coronary syndrome. Thromb Haemost 2010; 104: 976-83.

32. Schoenfeld H, Pruss A, Keller M, et al. Lyophilised plasma: evaluation of clotting factor activity over 6 days after reconstitution for transfusion. $J$ Clin Pathol 2010; 63: 726-30.

33. Steil L, Thiele T, Hammer E, et al. Proteomic characterization of freeze-dried human plasma: providing treatment of bleeding disorders without the need for a cold chain. Transfusion 2008; 48: 2356-63.

34. Samama MM, Contant G, Spiro TE, et al. Evaluation of the prothrombin time for measuring rivaroxaban plasma concentrations using calibrators and controls: results of a multicenter field trial. Clin Appl Thromb Hemost 2012; 18: 150-8.

35. Martinaud C, Civadier C, Ausset S, et al. In vitro hemostatic properties of French lyophilized plasma. Anesthesiology 2012; 117: 339-46.

36. Baglin T, Hillarp A, Tripodi A, et al. Measuring oral direct inhibitors (ODIs) of thrombin and factor Xa: A recommendation from the Subcommittee on Control of Anticoagulation of the Scientific and Standardisation Committee of the International Society on Thrombosis and Haemostasis. J Thromb Haemost 2013; 11: 756-60.

37. Cushman M, Lim W, Zakai N. American Society of Hematology 2011 Clinical Practice Guide on Anticoagulant Dosing and Management of Anticoagulant-Associated Bleeding Complications in Adults. Washington: American Society of Hematology; 2011. http://www.hematology. org/Clinicians/Guidelines-Quality/Quick-Ref/525.

38. Ageno W, Gallus AS, Wittkowsky A, et al. Oral anticoagulant therapy: Antithrombotic Therapy and Prevention of Thrombosis, 9th ed.: American College of Chest Physicians Evidence-Based Clinical Practice Guidelines. Chest 2012; 141(2 Suppl): e44-88S.

39. Cuker A, Siegal D. Monitoring and reversal of direct oral anticoagulants. Hematol Am Soc Hematol Educ Progr 2015; 2015: 117-24.

40. Heidbuchel H, Verhamme P, Alings M, et al. European Heart Rhythm Association Practical Guide on the use of new oral anticoagulants in patients with non-valvular atrial fibrillation. Europace 2013; 15: $625-51$.

41. Dale BJ, Chan NC, Eikelboom JW. Laboratory measurement of the direct oral anticoagulants. Br J Haematol 2016; 172: 315-36. 